\title{
Interaction of Titanium Dioxide Nanoparticles and Blood Serum of Patients with Bronchial Asthma and Myasthenia Gravis
}

\author{
Elena Arkhipova ${ }^{1}$, Irina Alchinova ${ }^{1}$, Alexander Sanadze ${ }^{2}$, Leonid Goldenberg $^{3}$, Mikhail Karganov $^{1}$ \\ ${ }^{1}$ Institute of General Pathology and Pathophysiology of Russian Academy of Medical Sciences, Laboratory of Physicochemical and \\ Ecological Pathophysiology, Moscow, Russia \\ ${ }^{2}$ SFHI Municipal Clinical Hospital No. 51, Moscow City Health Department, Moscow Myasthenia Center, Moscow, Russia \\ ${ }^{3}$ Plasmachem GmbH, Berlin, Germany
}

Email address:

mkarganov@mail.ru (M. Karganov)

\section{To cite this article:}

Elena Arkhipova, Irina Alchinova, Alexander Sanadze, Leonid Goldenberg, Mikhail Karganov. Interaction of Titanium Dioxide Nanoparticles and Blood Serum of Patients with Bronchial Asthma and Myasthenia Gravis. American Journal of Clinical and Experimental Medicine.

Vol. 3, No. 3, 2015, pp. 128-132. doi: 10.11648/j.ajcem.20150303.20

\begin{abstract}
The interest to nanoobjects is mainly attracted by the fact that their properties (and also biological in particular interest to the project) differ drastically from the properties of either bulk material or constituent molecules. The fundamental task is correlation between structure of the nanoparticles and their interaction with surrounding media. The aim of project is a study of interaction of titanium dioxide nanoparticles and blood serum of patients with verified myasthenia and allergy. Laser correlation spectroscopy (LCS) allows fast and easy hydrodynamic diameter determination in a broad range from nanometer to hundred micrometers, which is an advantage in comparison to other technique for a mixture of nano and microobjects of very different sizes, which constitutes biological liquids. LCS measurement of titanium dioxide nanoparticles specimen showed that main part of light scattering is due to particles with radial size 4.64-8.42 nm. LCS and other physical methods measurement data are coincided. Comparison of LC-histograms of blood serum revealed increased contribution of $4.64-15.28 \mathrm{~nm}$ particles into light scattering in bronchial asthma patients and 404.65 - $1794.35 \mathrm{~nm}$ particles in myasthenia gravis patients. Interaction between titanium dioxide nanoparticles, stabilized by phosphoric acid, and blood serum results to albumin- and globulin-containing complexes formation and precipitation. Interaction between titanium dioxide nanoparticles, stabilized by phosphoric acid, and blood serum results to albumin- and globulin-containing complexes formation and precipitation. Interaction between titanium dioxide nanoparticles and myasthenia patients blood serum results to removing of considerable part (40\%) of circulating immune complexes. TiO2 nanoparticles under interaction with asthma patients blood serum form complexes with small size proteins. LCS analysis confirmed removal of particles corresponding by their size to the major serum proteins and redistribution towards larger particles presented by glycolipids, glycoproteins, and antigen-antibody complexes.
\end{abstract}

Keywords: Autoimmune Complexes, Bronchial Asthma, Laser Correlation Spectroscopy, Myasthenia Gravis, Nanoparticles, Titanium Dioxide

\section{Introduction}

Modern level of scientific and technical progress implies the appearance of new materials with new properties and new applications of well-known technologies. Creation and application of materials with particle size of 9-10 $\mu$ became now possible [1]. The use of such materials in metallurgy, automobile industry, etc. becomes a commonplace, whereas in medicine it is still a novelty.

Nanopowders consist of particles $\leq 100 \mathrm{~nm}$ in diameter that due to their small size exhibit high biological activity and high penetration capacity. In the cell, nanoparticles can interact with its structural components and induce their damage. Nanoparticles can catalyze the formation of toxic agents in the body. Previous studies demonstrated toxic effects of nanopowders on laboratory animals [2] and their cytotoxic effects on tumor and bacterial cells. At the same time, nanopowders due to their antibacterial activity accelerate (by 1.5 times) healing of wounds and burns [3].

Titanium and titanium oxide are biocompatible materials. 
Here we studied the interaction of blood serum with titanium oxide nanoparticles. This implies the choice of optimal conditions, demonstration of this interaction, and evaluation of the direction of the induced processes.

\section{Materials and Methods}

We used standard serum prepared from the blood of conventionally healthy donors (Abbot, USA) and serum samples from patients with verified myasthenia gravis (10 patients) and bronchial asthma (20 patients). These diseases have autoimmune nature, which implies elevated content of autoantibodies.

Myasthenia gravis was diagnosed on the basis of clinical examination and electromyography results and the data of pharmacological tests with proserine (neostigmine) and Kalymin-Forte (pyridostigmine bromide), patients (male and female) with the mean age $41.7 \pm 7.5 ; \mathrm{M} \pm \delta$. According to current concept of myasthenia gravis pathogenesis, autoimmune aggression towards certain molecular structures of the skeletal muscle and neuromuscular junction is the leading pathophysiological factor responsible for the development of clinical manifestations of this disease. We have previously demonstrated that the contribution of immunoglobulin fraction into light scattering increased in patients with mild myasthenia gravis, while in patients with severe disease, autoimmune components predominated..

Bronchial asthma (BA) was diagnosed on the basis of the collection of anamnestic data, clinical examination, of respiratory function and laboratory tests, patients (male and female) with the mean age $43.4 \pm 5.4 ; \mathrm{M} \pm \delta$. Differences in the distribution of subcellular particles in the serum and urine of adult BA patients and healthy individuals have been demonstrated [4]. The observed integral metabolic shifts suggest that LCS can be applied for monitoring of BA patients, evaluation of treatment effectiveness, and detection of risk groups.

All tests were performed in accordance with Articles 5, 6 and 7 of Universal Declaration on Bioethics and Human Rights after written consent was obtained from patients.

Titanium oxide nanoparticles were provided by Institut Angewandte Polymerforschung (Germany). They had a diameter of $20 \mathrm{~nm}$, were stabilized with phosphoric acid, and represented a white powder. The properties of these nanoparticles during their interaction with biological fluids were never studied. Upon mixing with physiological saline, the nanoparticles form a transparent suspension. Since these nanoparticles are a new experimental object in biological studies, their working concentration was determined empirically and was $7.8 \mathrm{mg} / \mathrm{ml}$.

The processes accompanying the interaction of nanoparticles with serum were analyzed by the method of LCS. The method is based on changes in spectral characteristics of monochromatic coherent radiation of $\mathrm{He}-\mathrm{Ne}$ laser caused by light scattering in a disperse system (plasma, blood serum, urine, oropharyngeal lavage fluid) [5]. The results represent a histogram, where ordinate shows particle contribution into light scattering in percents and abscissa shows particle size in nanometers [6, 7].

Subfraction composition of biological fluids was recorded on a laser correlation spectrometer (LCS-03-INTOKS) approved by Committee for New Medical Equipment, Ministry of Health of the Russian Federation, for measuring microparticle size in biological fluids (Certificate RU.C. 39.003. A N 5381).

The sample $(0.2 \mathrm{~mL})$ was placed in a cuvette of an LCS spectrometer. Measurements were performed at a frequency of $16 \mathrm{kHz}$ (2000 accumulations). Spectrum regularization was performed using a nonlinear scale (Spectrometer and Blood software supplied with the spectrometer). Parameters of the reconstructed distribution appreciably approximate to actual values. The required information on the distribution shape can be obtained from experimental curves with noise level of $0.5-1 \%$.

In the given algorithm, the regularization parameter is determined on the basis of known error of experimental data. It is known that the major contribution into light scattering is made by proteins and their complexes that have spherical shape. The hydrodynamic radius of a spherical scattering particle somewhat surpasses the geometrical radius of a dry particle due to the formation of a shell consisting of solvent molecules. For globular proteins, the hydrodynamic size is close to the diameter of the macromolecule. For non-spherical particles, the contribution of orientation dynamics (and conformation dynamics for deformable particles) is significant. Therefore, the hydrodynamic size of non-spherical protein molecules is a rather rough parameter. At the same time, the coefficient of translation diffusion is an objective physical characteristic of the protein particle reflecting structural changes in macromolecules: aggregation, conformational transitions, enzyme-substrate interactions etc.

It should be noted that light scattering capacity of high-molecular-weight particles is higher than that of low-molecular-weight particles. Therefore, the method is more sensitive to large objects. In case of relatively high concentration, they can shield the low-molecular-weight fractions in LC spectrum.

The total protein content was measured by the method of Bradford [8]. The products of serum interaction with titanium oxide nanoparticles were lyophilized on Alpha I-5 lyophilizer («Martin Christ», Germany).

\section{Results and Discussion}

LCS analysis of the solution of titanium oxide nanoparticles showed that the main contribution into light scattering was made by particles with a radius of $4.64-8.42 \mathrm{~nm}$, which corresponded to the preparation specification (particle radius $10 \mathrm{~nm}$ ). Moreover, minor contribution into light scattering was made by $37.34-50.30 \mathrm{~nm}$ and $300.41-545.06 \mathrm{~nm}$ particles that probably appeared due to particle agglomeration. The LCS data on particle size agree with the results of physical tests (Fig. 1). 

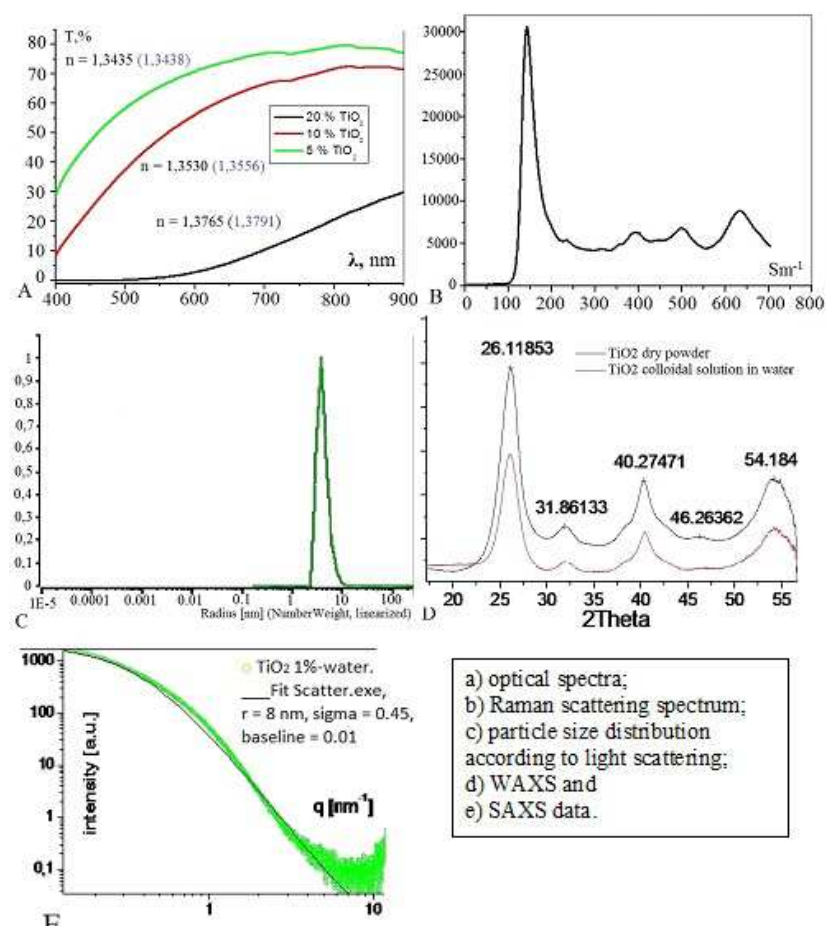

a) optical spectra;
b) Raman scattering spectrum;
c) particle size distribution
according to light scattering;
d) WAXS and
e) SAXS data.

Figure 1. Evaluation of the size and properties of titanium oxide nanoparticles by various physical methods.

The ratio of particles in the serum of patients with verified pathologies differed from the standard serum histogram: we observed predominance of $20.58-67.75 \mathrm{~nm}$ particles in patients and $122.92-300.41 \mathrm{~nm}$ particles in the standard serum (Fig. 2).

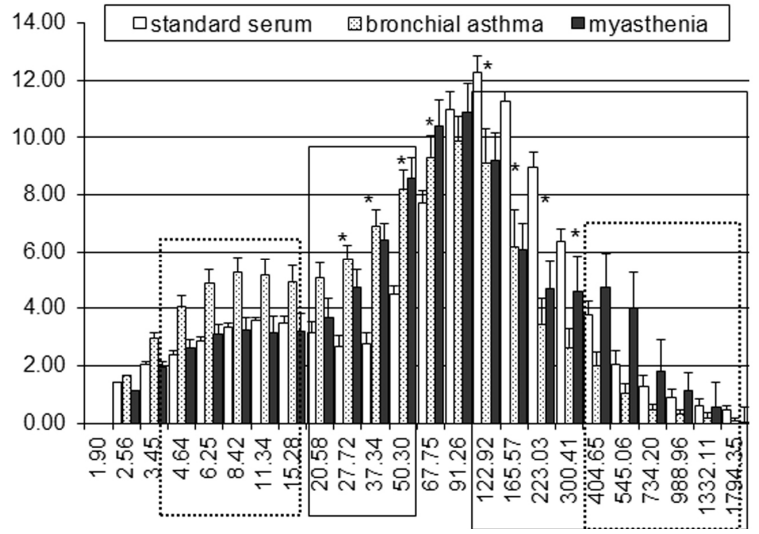

Figure. 2. LC-histogram of standard serum and serum samples from patients with verified pathologies. Abscissa: particle size, $\mathrm{nm}$; ordinate: contribution into light scattering, \%.*-comparison of standard serum and serum from myasthenia patient, - comparison of standard serum and serum from bronchial asthma patient, :........-- comparison of serum from myasthenia patient and bronchial asthma patient, Mann-Whitney test, $p$ $<0.05$.

Comparison of LC-histograms of blood serum revealed increased contribution of $4.64-15.28 \mathrm{~nm}$ particles into light scattering in bronchial asthma patients and 404.65 - 1794.35 $\mathrm{nm}$ particles in myasthenia gravis patients. This can be explained by pronounced inflammatory process accompanying bronchial asthma, in particular by the effects of complexes of cytokines and other markers of the infectious and allergic process.

Addition of nanoparticles to the serum was followed by the appearance of a white precipitate. We hypothesized that nanoparticles nonspecifically bound with proteins, which, on the one hand, led to elimination of small proteins, but on the other hand, complexes that could not be fully precipitated by centrifugation were still present in the serum.

LCS analysis confirmed removal of particles corresponding by their size to the major serum proteins and redistribution towards larger particles presented by glycolipids, glycoproteins, and antigen-antibody complexes.

LC-histograms presented in Figs. 3-5 reflect similarity of these processes.

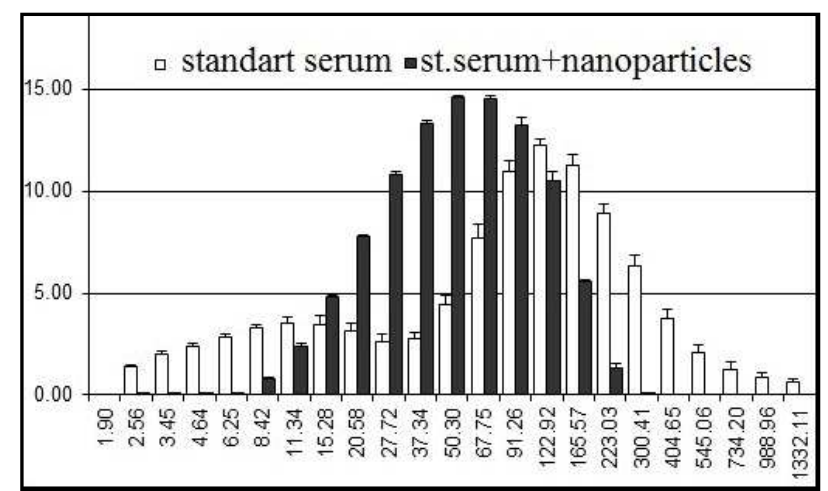

Figure 3. LC-histograms of standard serum: before and after interaction with titanium oxide nanoparticles. Axes as in Fig. 2.

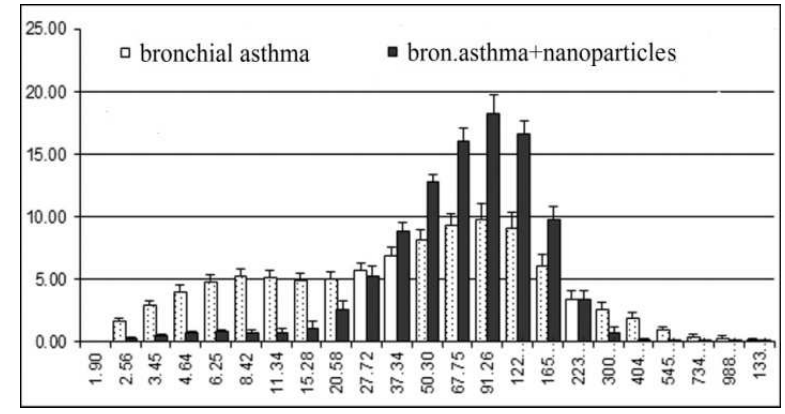

Figure 4. LC-histograms of blood serum from patients with bronchial asthma: before and after interaction with titanium oxide nanoparticles. Axes asin Fig. 2. $: \vdots: \vdots$ - significant differences, Mann-Whitney test, $p<0.05$.

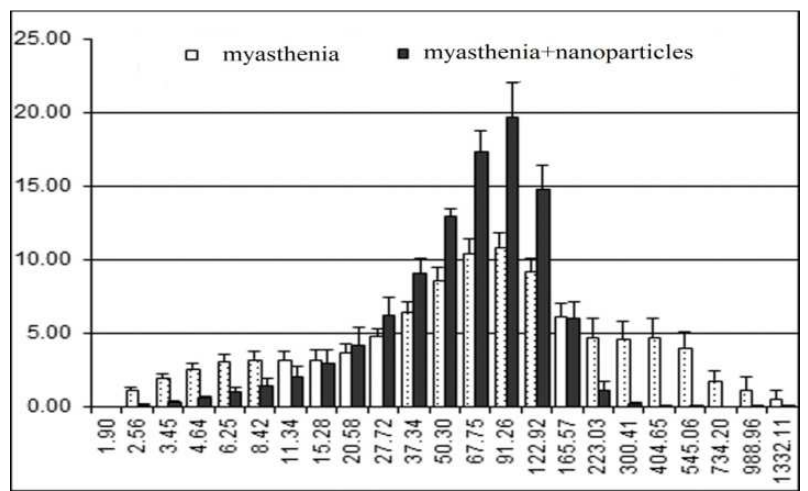

Figure. 5. LC-histograms of blood serum from patients with myasthenia gravis: before and after interaction with titanium oxide nanoparticles. Axes as in Fig. 2 : $2: \vdots$ significant differences, Mann-Whitney test, $p<0.05$. 
The contribution of particles $>223 \mathrm{~nm}$ that serve as the marker of autoimmunization decreased in the serum from patients with asthma, and even more so in the serum of myasthenia gravis patients.

For evaluation of the contribution of pathologies accompanying BA into metabolic homeostasis we performed LC analysis of urine and blood samples from patients with BA alone and patients with concomitant perennial allergic rhinitis. No significant changes in the distribution of light-scattering particles were revealed. This method was used by us in the study performed on a nuclear fuel plant for detection of groups at risk of hematological diseases and evaluation of the severity and prognosis of the course of these diseases on the basis of the detected metabolic shifts. Basing on the increase (or decrease) in the percent contribution of particles of this or that fraction into light scattering, the proposed semiotic classification of serum/plasma samples including 8 shifts of various directions in homeostasis and humoral immunity: intoxication-, catabolic-, dystrophic-, allergic-, autoimmune-like, and normological spectra and 2 types of mixed spectra. We evaluated the distribution of subcellular components of biological fluids from patients with hematological diseases (iron-deficient and B12-deficient anemias, lymphogranulomatosis, multiple myelomas, chronic lymphocytic leukemia, chronic myelocytic leukemia, thrombocytemia, polycytemia). Thus, a risk group for hematological diseases (anemia, $\mathrm{n}=11$ and erythremia, $\mathrm{n}=5$ ) was determined. The validity of the risk group formation was confirmed by standard hematological studies.

LCS analysis of blood serum and urine and standard clinical blood testing for eosinophil count detected a risk group for allergic diseases (5 women and 8 men). This group included individuals with moderate or pronounced allergic shifts and mixed shifts with the allergic component. Since the target was detection of the risk group by the brochopulmonary pathologies, further functional testing revealed reduced vital lung capacity in 5 examinees. The data were processed using an expert system. The core of the expert system is formed by a program executing autonomic evaluation of the results and database filling and management. It is based on relational database in Microsoft Access format (mdb). The expert system allows scoring the measured parameters using the hypo/hyperfunction scale taking into account the "individual norm" calculated from partial correlations and identification of the most important systems of sanogenesis and the source of strain. The expert system in the automatic mode provides parameters of electrical activity of the heart, heart rhythm, blood pressure, respiration, and psychomotor activity on the basis of patterns, spectral characteristics, transition processes, kinematograms, etc.; sex- and age-dependent statistical relationships; and takes into account intra- and inter-systemic nature of interactions between functional systems of the organism. Tiffeneau index was reduced in 4 individuals; this parameter is more important for evaluation of allegric bronchopulmonary pathologies. In 2 examinees, reduced Tiffeneau index was associated with increased relative content of eosinophils [9].
To confirm elimination of proteins from the serum, we measured protein content by the method of Bradford [8]. Upon contact with nanoparticles, up to $40 \%$ proteins were removed from the blood serum of patients and up to $70 \%$ proteins from standard serum. This was determined by protein availability, rather than its absolute content. The standard serum is prepared from the blood of healthy people and is characterized by strict proportion between the main components. The blood of patients with autoimmune diseases is characterized by the presence of large complexes and accessibility of various proteins can be reduced due to their binding with other substances of conformation changes.

The weight of lyophilized precipitates obtained after interaction of serum components with nanoparticles suggests that it consists of not only nanoparticles, but represents a complex of nanoparticles with serum proteins.

Thus, incubation of serum samples with titanium oxide nanoparticles stabilized with phosphoric acid led to the formation and precipitation of complexes consisting of nanoparticles and serum albumins and globulins. The interaction of titanium dioxide nanoparticles with blood serum from myasthenia patients resulted in removal of a great amount $(40 \%)$ of circulating immune complexes. In the serum of patients with bronchial asthma, titanium dioxide nanoparticles primarily bind small proteins. As a result of the interaction of serum proteins with the nanoparticles on the last form what is known "protein crown" [10.]

There is every reason to believe that nanoscale particles will be in the focus of interest for a long time, because they occupy an intermediate position between the atomic and molecular state and condensed state of the matter. The fundamental objectives are analysis of their electronic structure, interaction with the environment, surface structure and its influence on nanoparticle stability, and catalytic properties (influence on the rate of various chemical reactions).

It should be noted that measurement of nanoparticle radius remains a difficult problem. Most of the existing methods either do not allow measurements in the range of nanostructures, or are very difficult. However, the use of dynamic light scattering methods and solution of the inverse problem by using regularization approach allowed us to construct the particle size distribution from the spectrum of fluctuation of the light scattered on these particles. The range of this method is from nanometers to tens and even hundreds of micrometers (5-6 orders of magnitude), which is unachievable for other methods $[11,12]$. The obtained results suggest that the method of laser correlation spectroscopy is an adequate and convenient tool for the study of interaction of nanoobjects with components of biological fluids.

The detected binding of nanoparticles with large immune complexes typical of severe forms of myasthenia gravis [13] is of undoubted practical importance. At present, plasmapheresis is used for removal of circulating immune complexes from the blood in myasthenia patients. The detected interaction of nanoparticles with serum components opens new vistas for the development of nanotechnologies for the therapy of this disabling pathology. 


\section{References}

[1] W.H.De Jong, P.JA Borm, "Drug delivery and nanoparticles: Applications and hazards," International Journal of Nanomedicine, 2008, 3(2), pp.133-149.

[2] P. Thevenot, Jai Cho, D. Wavhal, R. B. Timmons, L. Tang, "Surface chemistry influences cancer killing effect of $\mathrm{TiO} 2$ nanoparticles," Nanomedicine: NBM, 2008, 4:226-236. doi:10.1016/j.nano.2008.04.001.

[3] Bae Y. H. and Park K., "Targeted drug delivery to tumors: Myths, reality and possibility," J. Controlled Release, 2012; 153(3), pp. 198-205.

[4] Ezhova O., Klebnikova N., Alchinova I., Arkhipova E., Torshin V., Karganov M., "New methods of laboratory diagnosis and monitoring the effectiveness of allergic diseases," Vestnik RUDN. Ser. Medicina, 2008, 3:52-58. (in Russian)

[5] Karganov M., Kiselyov M., Komarov G., Kuinzhi N., Kurneshova L., Kuchma V., etc. "Polysystem sanogenetichesky monitoring," Moscow, MIPKRO, 2001, 344 p. (in Russian)

[6] Titov V.N., Karganov M.Yu., Rotenko A.A., Dmitriyev V.A., Alchinova I.B., Arkhipova E.N., "Biological functions and biological reactions. laser correlation spectroscopy in an assessment of purity of the intercellular environment functions of an endoekologiya (lecture)," Klinicheskaja laboratornaja diagnostika, 2009, vol. 6, pp. 21-36. (in Russian)

[7] Karganov M., Alchinova I., Arkhipova E., Skalny A.V., "Laser Correlation Spectroscopy: Nutritional, Ecological and Toxic Aspects," In: "Biophysics". A.N. Misra ed.- InTech. 2012, ISBN 978-953-51-0376-9: pp.1-16.

[8] Bradford M.M., "A rapid and sensitive method for the quantitation of microgram quantities of protein utilizing the principle of protein-dye binding," Anal. Biochem, 1976, vol. 72 , pp. $248-254$.

[9] Alchinova I., Arkhipova E., Cherepov A., Karganov M., "Polysystemic monitoring of nuclear plant stuff and risk group revealing," In: Risk assessment and management. Zhang Zhiyong ed., 2012, Academy Publish, USA. ISBN: 978-0-9835850-2-2. P. 193-199.

[10] Mirshafiee V., Mahmoudi M., Lou K., Cheng J. and Kraft M. L., "Protein corona significantly reduces active targeting yield," Chem. Commun, 2013, vol. 49, pp. 2557-9. doi: $10.1039 / \mathrm{c} 3 \mathrm{cc} 37307 \mathrm{j}$.

[11] Zhdanov R.I., Strazhevskaja N.B., Shmyrina A.S., Krylov A.S., Zarubina T.V., Karganov M.Yu. et al., "Instability of genomic DNA and change of composition of fatty acids of the DNA-connected lipids of a spleen of rats at the folat-induced gipergomotsisteinemiya," Patogenez. 2006, 4 (4), pp. 82-92. (in Russian)

[12] Piruzjan L.A., Kovalev I.E., Kovaleva V.L., Tjumenceva E.S., Balabolkin I.I., Karganov M.Yu. et al., "Laser correlation spectroscopy of macromolecular complexes in blood serum as an effective method of an assessment of a course of a disease of bronchial asthma at children," Doklady Akademii nauk, 2004, 395(6), pp. 832-836. (in Russian)

[13] Alchinova, E. Arkhipova, D. Sidnev, A. Sanadze, S. Dedaev, and M. Karganov, "Comparative Analysis of the Informative Value of Radioimmunoassay and Laser Correlation Spectroscopy in Myasthenia Gravis, " ISRN Immunology, vol. 2014, Article ID 718393, 5 pages, 2014.doi: $10.1155 / 2014 / 718393$ 